\title{
Fluorinated 3,4-Dihydro-2H-1,4-Benzothiazin- 1,1-Dioxide Derivatives with Antiarrhythmic and Hypertensive Effects
}

\author{
Bryzgalov $\mathrm{AO}^{1 *}$, Yakovleva TA², Politanskaya $\mathrm{LV}^{1}$, Bagryanskaya IY', and Tolstikova ${ }^{1,2}$ \\ ${ }^{1}$ NN Vorozhtsov Novosibirsk Institute of Organic Chemistry, Russia \\ ${ }^{2}$ Novosibirsk State University, Russia
}

*Corresponding author: Bryzgalov AO, NN Vorozhtsov Novosibirsk Institute of Organic Chemistry, Russia.

To Cite This Article: Bryzgalov AO, Yakovleva TA, Politanskaya LV, Bagryanskaya IY, Tolstikova. Fluorinated 3,4-Dihydro-2H-1,4-Benzothiazin1,1-Dioxide Derivatives with Antiarrhythmic and Hypertensive Effects. Am J Biomed Sci \& Res. 2021 - 13(4). AJBSR.MS.ID.001882.

DOI: 10.34297/AJBSR.2021.13.001882.

Received: 眥 June 01, 2021; Published: 畊 July 09, 2021

\begin{abstract}
A series of fluorinated 3,4-dihydro-2H-1,4-benzothiazine derivatives was synthesized starting from pentafluorobenzoic acid and 2-mercaptoethanol via aromatic nucleophilic substitution of fluorine atoms. They were characterized by spectral methods, X-ray diffraction analysis, and screened for the cardiotropic activity in vivo on mature male rats of line Wistar. The presence of a possible antiarrhythmic activity in the selected derivatives was tested, as was the effect on blood pressure in widely accepted models of arrhythmia. It was found that this series of chemical compounds has an antiarrhythmic effect and causes a monotonic increase in blood pressure within 3-5min. As the most promising agents were selected 5,7 difluoro-3,4-dihydro-2H-benzo[1,4]thiazine-1,1-dioxide derivatives 3c and 5, containing a cyclopentylamine fragment at position 8.
\end{abstract}

Keywords: Fluorinated benzothiazines; Antiarrhythmic activity; Hypertensive effect

\section{Introduction}

Arrhythmias account for substantial cardiac morbidity and mortality in the past few decades [1]. Although some arrhythmias may appear benign or potentially dangerous, most sudden cardiac arrests are a direct result of ventricular tachycardia and/or ventricular fibrillation [2,3]. Although conventional antiarrhythmic drugs have been reported to be effective in preventing arrhythmias, they themselves can cause lethal arrhythmias in some clinical conditions. It is known that electrical defibrillation is the only effective way to treat ventricular fibrillation [4]. Currently, the shock strength required to successfully defibrillate is still high. A few studies have been aimed at lowering the defibrillation threshold. One of the means to decrease the defibrillation threshold is to combine defibrillation with medication [5]. Both pharmacological and nonpharmacological interventions for preventing these lethal arrhythmias have been widely investigated. At the same time, the search for low-toxicity highly effective drugs remains very relevant in modern cardiology.

Hypotension is one of the problems in the treatment of cardiovascular diseases. Most studies deal with orthostatic hypotension [6,7] whereas chronic arterial hypotension is less investigated. The prevalence of orthostatic hypotension is age dependent, ranging from $5 \%$ in patients $<50$ years of age to $30 \%$ in those older than 70 years [8,9]. Orthostatic hypotension is the second most common etiology of syncope and is seen in approximately $15 \%$ of syncope cases [10].

The presence of cardiotropic activity in 3,4-dihydro-2 $H$-1,4benzothiazin-1,1-dioxide derivatives has been shown. It has been suggested that benzothiazine derivatives may have antiarrhythmic activity and affect the cardiovascular system (by increasing or decreasing blood pressure). Considerable progress has been made 
in the development of antiarrhythmic drugs of different chemical classes and drugs that normalize blood pressure. Meanwhile, to improve the treatment and prevention of various cardiovascular diseases, it is necessary to create new drugs that are effective in much smaller doses than the existing drugs are. In addition, today on the market, there are virtually no drugs that can cause a gradual increase in blood pressure in patients with hypotensive disorders without causing arrhythmia (not to mention preventing it). 1,4-Benzothiazine derivatives, including 3,4-dihydro- $2 H$-1,4benzothiazines, have a wide variety of biological activities and are used in drug design [11,12]. Therefore, this type of scaffold is important for medicinal chemistry. Fluorination of a heterocyclic scaffold is known to enhance the bioactivity of complicated organic compounds.

\section{Materials and Methods}

\section{Animals and Experimental Procedures}

\section{In vivo experiments}

The pharmacological experiments were carried out on several groups of mature male rats of the Wistar strain in accordance with The Guideline for the Care and Use of Laboratory Animals. All the experiments were performed following the guidelines of the European Convention for the Protection of Vertebrate Animals Used for Experimental and Other Scientific Purposes (ethical approval for the animal experiments № P-13-012121-15 01.15.2021). Each group of rats contained 10 animals. The rats, weighing 190-220g, age 2-3 months, were obtained from the Institute of Cytology and Genetics, the Siberian Branch of the Russian Academy of Sciences (Novosibirsk, Russia). The rats were anesthetized with sodium thiopental $(0.12 \mathrm{mg} / \mathrm{kg})$ by intraperitoneal administration. After the induction of anesthesia, the animals were restrained, and an Electrocardiogram (ECG) was recorded. For this purpose, three electrodes were affixed to the body of the rats. The electrodes were connected to an ECG amplifier: the V75-11 ECG Isolated Amplifier (Coulbourn Instruments; USA). It performs all the standard multilead ECG monitoring functions under the control of LabView 6.1 software. Left ventricular pressure levels were measured with a high-fidelity low-cost blood pressure transducer (V94-21, Coulbourn Instruments; USA) placed in the left ventricle via the right carotid artery. Arrhythmia was induced by either one-step intravenous injection of a $10 \% \mathrm{CaCl}_{2}$ solution in a dose of $250 \mathrm{mg} /$ $\mathrm{kg}$ or an epinephrine injection at $0.3 \mathrm{mg} / \mathrm{kg}$ into rats anesthetized with sodium thiopental $(0.12 \mathrm{mg} / \mathrm{kg}$, intraperitoneally). These doses of the arrhythmogenesis are lethal (100\% lethal dose: LD100) for rats. Tested agents were mixed with Tween 66, the mixtures were diluted with physiological saline, and the resulting formulations were injected into the vena femoralis (the same site in all the rats). This route of administration allows to block acute arrhythmia directly. After the induction of anesthesia, the animals were restrained, and an ECG was recorded via a second standard lead for 10min. Durations of RR, PQ, QRS, QT, and P wave intervals were estimated together with amplitudes of the $\mathrm{P}, \mathrm{T}$, and $\mathrm{R}$ waves. Statistical analysis was performed in the STATISTICA 6.0 software and in software developed at the Novosibirsk Institute of Organic Chemistry for calculating ECG parameters.

\section{Ex vivo experiments}

Right atria from Wistar rats were prepared as described previously [10]. Briefly, the animals were killed by $\mathrm{CO}_{2}$ asphyxiation, and the right atria were quickly excised and immersed in RingerLocke's solution [approximate concentrations in grams per liter: $\mathrm{NaCl}$ (9.0-9.5), $\mathrm{KCl}$ (0.20-0.42), $\mathrm{CaCl}_{2}$ (anhydrous, 0.20$0.24), \mathrm{NaHCO}_{3}(0.1-0.3)$, and glucose (1.0-2.5)]. The right atria with sinus cardiac pacemakers were dissected and mounted in isolated organ baths filled with $10 \mathrm{~mL}$ of Ringer-Locke's solution maintained at $37^{\circ} \mathrm{C}$ and aerated with $95 \% \mathrm{O}_{2}$ and $5 \% \mathrm{CO}_{2}$. To achieve a steady spontaneous tone level, an initial tension of $1 \mathrm{~g}$ was applied. Contractions were measured isometrically with a force-displacement transducer (PanLab s.l., Spain) and recorded by Isolated Organs Data Acquisition software (Protowin; Panlab Technology for Bioresearch, Spain). Tissues were allowed to stabilize for $20 \mathrm{~min}$, whereas the bathing solution was exchanged at 5 min intervals. After re-establishment of stable baseline tone, our compounds were added to the tissues $\left(10^{-6}\right.$ to $\left.10^{-3} \mathrm{M}\right)$.

\section{Chemistry}

\section{Synthetic approaches}

The synthesis of fluorinated 3,4-dihydro-2H-1,4-benzothiazine derivatives was carried out by a methodology previously developed in our laboratory $[13,14]$. Briefly, the synthesis of 1 was accomplished in four steps: by aromatic nucleophilic substitution of a fluorine atom in pentafluorobenzoic acid, cautious oxidation of sulfide to sulfoxide, a reaction of decarboxylation, and finally, by replacement of the hydroxyl group with a chlorine atom in the side chain as shown in Figure 1.

The heterocyclic compounds 2 and 4 [14] were prepared via the replacement of chlorine atom by the action of primary amines accompanied by intramolecular aromatic nucleophilic substitution of the ortho-fluorine atom in the ring. Then the synthesis of target compounds 3a-c and 5 were realized by the amino defluorination in 8 position of heterocycles 2 and 4 correspondingly (Figure 1). All derivatives were obtained in good yields (73-99\%). 

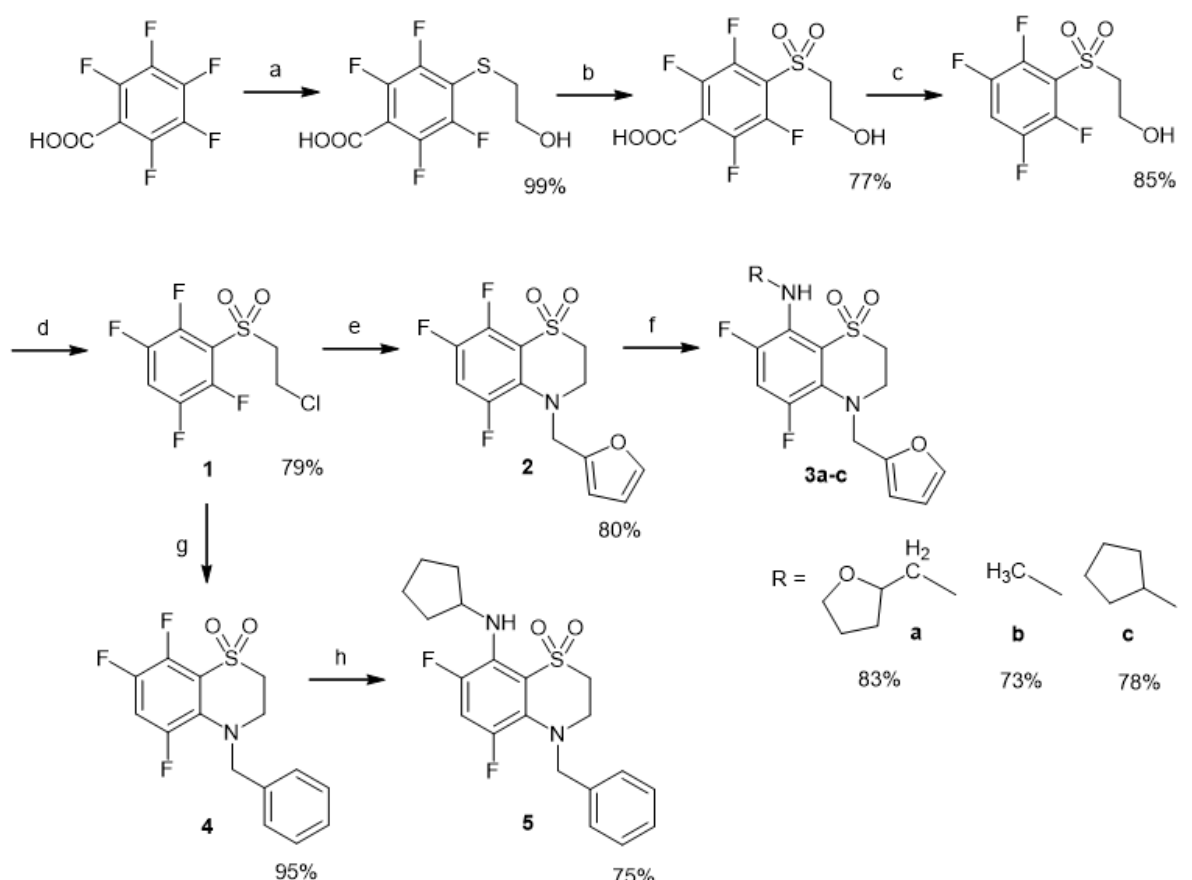

Figure 1: Reagents and conditions; (a) $\mathrm{HSCH}_{2} \mathrm{CH}_{2} \mathrm{OH}, \mathrm{Et}_{3} \mathrm{~N}, \mathrm{H}_{2} \mathrm{O}$, rt; (b) $\mathrm{H}_{2} \mathrm{O}_{2}, \mathrm{CF}_{3} \mathrm{COOH}$, rt; (c) $\mathrm{Et}_{3} \mathrm{~N}, \mathrm{MeCN}_{2}$, reflux; (d) $\mathrm{SOCl}$, $\mathrm{CHCl}_{3}$, reflux; (e)

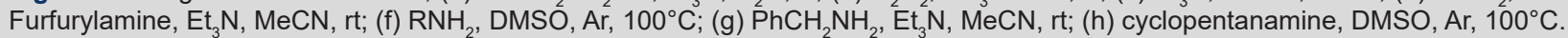
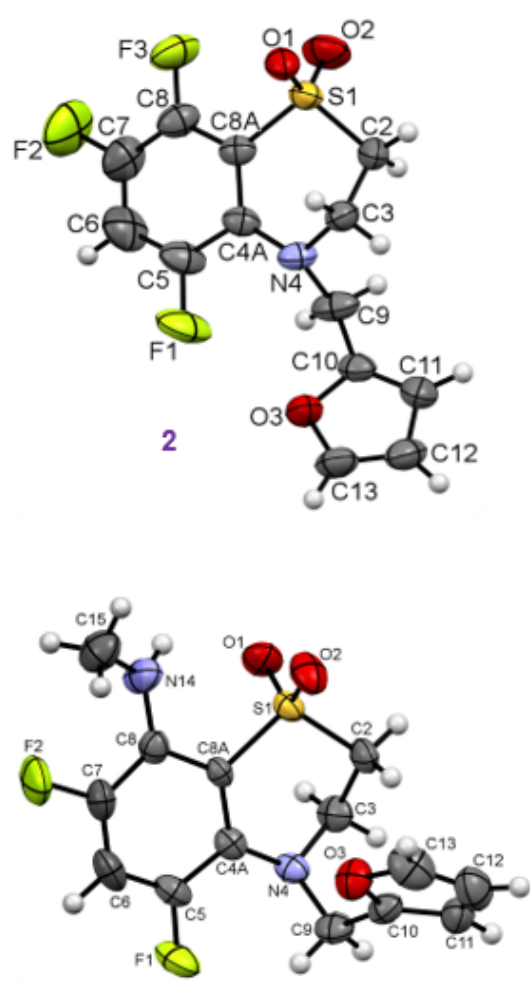

$3 \mathrm{~b}$

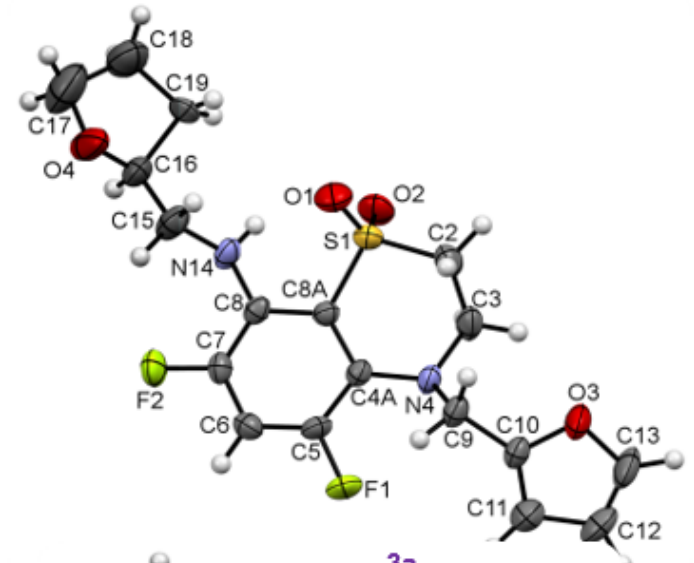

$3 a$

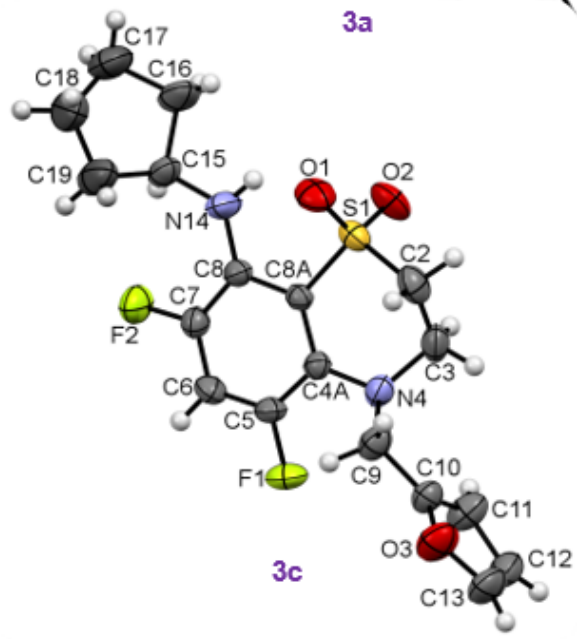

Figure 2: The crystal structures of 2 and 3a-c. 
The structures of all new products were confirmed by ${ }^{1} \mathrm{H},{ }^{19} \mathrm{~F}$, ${ }^{13} \mathrm{C}$ NMR; Infrared (IR) spectroscopy; and high-resolution mass spectrometry (HRMS). The molecular and crystal structures of 2 and 3a-c were solved by X-ray crystallography (Figure 2).

\section{Experimental Protocols}

\section{Chemistry}

\section{General}

All solvents and $\mathrm{SOCl}_{2}$ were purified using standard procedures. Commercially supplied pentafluorobenzoic acid, 2-mercaptoethanol, furfurylamine, tetrahydrofurfurylamine, cyclopentanamine, phenylmethanamine, methanamine (35\% aq. solution), $\mathrm{H}_{2} \mathrm{O}_{2}$ ( $40 \%$ aq. solution), trifluoroacetic acid were used without purification. The starting 3-(2-chloroethylsulfonyl)-1,2,4,5tetrafluorobenzene (1) was synthesized according to previously described methods [13-20]. Preparative TLC was performed on Merck precoated silica gel $60 \mathrm{PF}_{254}$ containing gypsum. Visualization of the developed chromatograms was performed by UV light. To obtain analytically pure samples, the solid synthesized compounds were crystallized from diethyl ether.

NMR spectra were recorded on a Bruker Avance-300 $\left(300.13 \mathrm{MHz}\right.$ for $1 \mathrm{H}$ and $282.37 \mathrm{MHz}$ for ${ }^{19} \mathrm{~F}$ ) and $\mathrm{DRX}-500$ $\left(500.13 \mathrm{MHz}\right.$ for ${ }^{1} \mathrm{H}, 125.76 \mathrm{MHz}$ for ${ }^{13} \mathrm{C}$ ) spectrometers. $\mathrm{CDCl}_{3}$ and acetone-d6 were used as solvents, with residual $\mathrm{CHCl}_{3}\left(\delta_{\mathrm{H}}=7.26\right)$ or $\mathrm{CDCl}_{3}\left(\delta_{\mathrm{C}}=77.0\right)$ and acetone $\left(\delta_{\mathrm{H}}=2.15\right)$ or acetone-d6 $\left(\delta_{\mathrm{C}}=28.6\right.$ and 205.0) being employed as internal standards. $\mathrm{C}_{6} \mathrm{~F}_{6}\left(\delta_{\mathrm{F}}=163.0\right)$ was used as external reference for recording the ${ }^{19} \mathrm{~F}$ NMR spectra. ${ }^{13} \mathrm{C}$ NMR spectra were recorded with $\mathrm{C}-\mathrm{H}$ spin decoupling. Masses of molecular ions were determined by HRMS on a DFS Thermo scientific instrument (EI, 70eV). Melting points were recorded on a Mettler-Toledo FP81 Thermosystem apparatus. The IR spectra were recorded on a Bruker Vector 22 spectrometer (KBr).

The structures of all new polyfluorinated compounds 2-5 prepared here were corroborated by their ${ }^{19} \mathrm{~F},{ }^{1} \mathrm{H}$ and ${ }^{13} \mathrm{C}$ NMR, high-resolution mass spectrometry, and IR-spectroscopy data (see SI). Signals in the NMR spectra of 2-5 were assigned based on spin coupling constants, which are typical for polyfluorinated benzenes [15-20].

\section{Synthetic procedures}

\section{4-Furfuryl-5,7,8-trifluoro-3,4-dihydro-2H-benzo[1,4]thiazine-}

\section{1,1-dioxide (2)}

To a solution of tetrafluorobenzene $1(1.9 \mathrm{~g}, 6.7 \mathrm{mmol})$ in MeCN $(50 \mathrm{~mL})$ furfurylamine $(1.0 \mathrm{~g}, 10 \mathrm{mmol})$ and $\mathrm{Et}_{3} \mathrm{~N}(3.5 \mathrm{~mL})$ were added. The reaction mixture was stirred at rt for $72 \mathrm{~h}$. Then the mixture was diluted with $\mathrm{H}_{2} \mathrm{O}(50 \mathrm{~mL})$ and extracted with $\mathrm{CH}_{2} \mathrm{Cl}_{2}$ $(2 \times 50 \mathrm{~mL})$. The combined organic layers were washed with $\mathrm{H}_{2} \mathrm{O}$
$(50 \mathrm{~mL})$ and dried $\left(\mathrm{MgSO}_{4}\right)$. After the evaporation of the solvent in vacuo, the crude product as oil was obtained.

White solid (recrystallized from ethanol); yield: 1.7g (80\%); mp 105.5-107.1 ${ }^{\circ} \mathrm{C}$. IR (KBr): 3132, 3055, 2995, 2936, 1636, 1490, 1458, 1363, 1349, 1314, 1302, 1241, 1182, 1129, 1087, 1020, 991, 961, 937, 892, 761, 741, 687, 601, 554, 496, $474 \mathrm{~cm}$ ${ }^{1}$. ${ }^{1} \mathrm{H}$ NMR $\left(300 \mathrm{MHz}, \mathrm{CDCl}_{3}\right): \delta=7.36\left(\mathrm{dd}, J\left(\mathrm{H}^{13}, \mathrm{H}^{12}\right)=1.9 \mathrm{~Hz}, 1 \mathrm{H}\right.$, $\left.\mathrm{H}^{13}\right), 7.09\left(\mathrm{~m}, \mathrm{~J}\left(\mathrm{H}^{6}, \mathrm{~F}^{5}\right)=12.1 \mathrm{~Hz}, \mathrm{~J}\left(\mathrm{H}^{6}, \mathrm{~F}^{7}\right)=9.5 \mathrm{~Hz}, \mathrm{~J}\left(\mathrm{H}^{6}, \mathrm{~F}^{8}\right)=7.3 \mathrm{~Hz}, 1 \mathrm{H}\right.$, $\left.\mathrm{H}^{6}\right), 6.31\left(\mathrm{dd}, \mathrm{J}\left(\mathrm{H}^{12}, \mathrm{H}^{11}\right)=3.3 \mathrm{~Hz}, J\left(\mathrm{H}^{12}, \mathrm{H}^{13}\right)=1.9 \mathrm{~Hz}, 1 \mathrm{H}, \mathrm{H}^{12}\right), 6.25(\mathrm{~d}$, $\left.\mathrm{J}\left(\mathrm{H}^{11}, \mathrm{H}^{12}\right)=3.3 \mathrm{~Hz}, 1 \mathrm{H}, \mathrm{H}^{11}\right), 4.46\left(\mathrm{~s}, 2 \mathrm{H}, \mathrm{H}^{9}\right), 3.86\left(\mathrm{tm}, \mathrm{J}\left(\mathrm{H}^{3}, \mathrm{H}^{2}\right)=5.6 \mathrm{~Hz}\right.$, $\left.2 \mathrm{H}, \mathrm{H}^{3}\right), 3.19\left(\mathrm{tm}, \mathrm{J}\left(\mathrm{H}^{2}, \mathrm{H}^{3}\right)=5.6 \mathrm{~Hz}, 2 \mathrm{H}, \mathrm{H}^{2}\right) .{ }^{13} \mathrm{C} \mathrm{NMR}\left(126 \mathrm{MHz}, \mathrm{CDCl}_{3}\right)$ : $\delta=149.9\left(\mathrm{~s}, \mathrm{C}^{10}\right), \quad 148.6\left(\mathrm{ddd},{ }^{1} \mathrm{~J}\left(\mathrm{C}^{5}, \mathrm{~F}^{5}\right)=249.1 \mathrm{~Hz}, \quad 3 \mathrm{~J}\left(\mathrm{C}^{5}, \mathrm{~F}^{7}\right)=8.1 \mathrm{~Hz}\right.$, $\left.\mathrm{C}^{5}\right), \quad 144.5\left(\mathrm{ddd}, \quad{ }^{1} \mathrm{~J}\left(\mathrm{C}^{8}, \mathrm{~F}^{8}\right)=255.6 \mathrm{~Hz}, \quad{ }^{2} \mathrm{~J}\left(\mathrm{C}^{8}, \mathrm{~F}^{7}\right)=14.7 \mathrm{~Hz}, \quad \mathrm{C}^{8}\right)$, $143.5\left(\mathrm{ddd},{ }^{1} \mathrm{~J}\left(\mathrm{C}^{7}, \mathrm{~F}^{7}\right)=249.4 \mathrm{~Hz},{ }^{2} \mathrm{~J}\left(\mathrm{C}^{7}, \mathrm{~F}^{8}\right)=13.8 \mathrm{~Hz},{ }^{3} \mathrm{~J}\left(\mathrm{C}^{7}, \mathrm{~F}^{5}\right)=11.4 \mathrm{~Hz}\right.$, $\left.\mathrm{C}^{7}\right), \quad 143.0\left(\mathrm{~s}, \mathrm{C}^{13}\right), \quad 130.9\left(\mathrm{dd},{ }^{2} \mathrm{~J}\left(\mathrm{C}^{4 \mathrm{a}}, \mathrm{F}^{5}\right)=11.9 \mathrm{~Hz}, \mathrm{C}^{4 \mathrm{a}}\right), \quad 120.7(\mathrm{dd}$, $\left.{ }^{2} \mathrm{~J}\left(\mathrm{C}^{8 \mathrm{a}}, \mathrm{F}^{8}\right)=13.0 \mathrm{~Hz}, \mathrm{C}^{8 \mathrm{a}}\right), 110.7\left(\mathrm{~s}, \mathrm{C}^{12}\right), 109.6\left(\mathrm{ddm},{ }^{2} \mathrm{~J}\left(\mathrm{C}^{6}, \mathrm{~F}^{7}\right)=27.1 \mathrm{~Hz}\right.$, $\left.{ }^{2} \mathrm{~J}\left(\mathrm{C}^{6}, \mathrm{~F}^{5}\right)=21.8 \mathrm{~Hz}, \mathrm{C}^{6}\right), 109.5\left(\mathrm{~s}, \mathrm{C}^{11}\right), 50.4\left(\mathrm{~d}, \mathrm{~J}\left(\mathrm{C}^{9}, \mathrm{~F}^{5}\right)=11.4 \mathrm{~Hz}, \mathrm{C}^{9}\right)$, 49.3(s, $\left.\mathrm{C}^{2}\right), \quad 47.3\left(\mathrm{~s}, \mathrm{C}^{3}\right) .{ }^{19} \mathrm{~F}$ NMR $\left(282 \mathrm{MHz}, \mathrm{CDCl}_{3}\right): \delta=-120.8(\mathrm{t}$, $\left.\mathrm{J}\left(\mathrm{F}^{5}, \mathrm{~F}^{8}\right) \approx \mathrm{J}\left(\mathrm{F}^{5}, \mathrm{H}^{6}\right)=12.8 \mathrm{~Hz}, \quad 1 \mathrm{~F}, \quad \mathrm{~F}^{5}\right), \quad-141.3 \quad\left(\mathrm{~m}, \quad J\left(\mathrm{~F}^{8}, \mathrm{~F}^{7}\right)=22.6 \mathrm{~Hz}\right.$, $\left.J\left(F^{8}, F^{5}\right)=13.5 \mathrm{~Hz}, J\left(F^{8}, H^{6}\right)=7.3 \mathrm{~Hz}, 1 \mathrm{~F}, \mathrm{~F}^{5}\right),-142.7\left(\mathrm{ddm}, J\left(\mathrm{~F}^{7}, \mathrm{~F}^{8}\right)=22.6 \mathrm{~Hz}\right.$, $\left.J\left(\mathrm{~F}^{7}, \mathrm{H}^{6}\right)=9.5 \mathrm{~Hz}, 1 \mathrm{~F}, \mathrm{~F}^{7}\right)$. HRMS(EI): $m / z[\mathrm{M}]^{+}$calcd for $\mathrm{C}_{13} \mathrm{H}_{10} \mathrm{~F}_{3} \mathrm{NO}_{3} \mathrm{~S}$ : 317.0328; found: 317.0325 .

\section{4-R-8-R'-5,7-Difluoro-3,4-dihydro-2H-benzo[1,4] thiazine-1,1- dioxide 3a-c and 5 General Procedure}

To a solution of trifluoroarene $(0.95 \mathrm{~g}$ for 2 and $0.98 \mathrm{~g}$ for 4 , $3.0 \mathrm{mmol})$ in DMSO (3mL) were added primary amine $\mathrm{RNH}_{2}(0.45 \mathrm{~g}$ for tetrahydrofurfurylamine, $0.38 \mathrm{~g}$ for phenylmethanamine, $1.2 \mathrm{~g}$ for methanamine ( $35 \%$ aq. solution), $4.5 \mathrm{mmol}$ ). The reaction mixture was stirred at $100^{\circ} \mathrm{C}$ under argon atmosphere for $48 \mathrm{~h}$. Then the mixture was diluted with $\mathrm{H}_{2} \mathrm{O}(50 \mathrm{~mL})$ and extracted with $\mathrm{Et}_{2} \mathrm{O}(2 \times 50 \mathrm{~mL})$. The combined organic layers were washed with $\mathrm{H}_{2} \mathrm{O}(50 \mathrm{~mL})$ and dried $\left(\mathrm{MgSO}_{4}\right)$. After the evaporation of the solvent in vacuo, the crude product as oil was obtained.

\section{4-Furfuryl-8-tetrahydrofurfurylamino-5,7-difluoro-3,4- dihydro-2H-benzo[1,4] thiazine-1,1-dioxide (3a)}

White solid (recrystallized from diethyl ether); yield: $0.99 \mathrm{~g}$ (83\%); mp 101.7-102.3 ${ }^{\circ} \mathrm{C}$. IR (KBr): 3396, 2976, 2937, 2872, $1601,1485,1452,1402,1360,1281,1230,1178,1122,1063$, 1016, 989, 955, 860, 818, 756, 715, 600, 571, 550, 472 $\mathrm{cm}^{-1} .{ }^{1} \mathrm{H}$ NMR $\left(500 \mathrm{MHz}, \mathrm{CDCl}_{3}\right): \delta=7.37\left(\mathrm{dd}, \mathrm{J}\left(\mathrm{H}^{13}, \mathrm{H}^{12}\right)=1.8 \mathrm{~Hz}, 1 \mathrm{H}, \mathrm{H}^{13}\right)$, $6.90\left(\mathrm{t}, J\left(\mathrm{H}^{6}, \mathrm{~F}^{5}\right) \approx J\left(\mathrm{H}^{6}, \mathrm{~F}^{7}\right)=12.1 \mathrm{~Hz}, 1 \mathrm{H}, \mathrm{H}^{6}\right), 6.31\left(\mathrm{dd}, \mathrm{J}\left(\mathrm{H}^{12}, \mathrm{H}^{11}\right)=3.2 \mathrm{~Hz}\right.$, $\left.\mathrm{J}\left(\mathrm{H}^{12}, \mathrm{H}^{13}\right)=1.8 \mathrm{~Hz}, 1 \mathrm{H}, \mathrm{H}^{12}\right), \quad 6.23\left(\mathrm{~d}, \quad \mathrm{~J}\left(\mathrm{H}^{11}, \mathrm{H}^{12}\right)=3.2 \mathrm{~Hz}, 1 \mathrm{H}, \mathrm{H}^{11}\right)$, $5.24\left(\mathrm{t}, 1 \mathrm{H}, \quad J\left(\mathrm{H}^{14}, \mathrm{H}^{15}\right)=5 \mathrm{~Hz}, \mathrm{H}^{14}[\mathrm{NH}]\right), 4.35\left(\mathrm{~s}, 2 \mathrm{H}, \mathrm{H}^{9}\right), 4.10(\mathrm{~m}$, $\left.1 \mathrm{H}, \mathrm{H}^{17}\right), 3.89\left(\mathrm{~m}, 1 \mathrm{H}, \mathrm{H}^{17}\right), 3.79-3.74\left(\mathrm{~m}, 3 \mathrm{H}, 2 \mathrm{H}^{3}+\mathrm{H}^{16}\right)$, 3.46$3.33\left(\mathrm{~m}, 2 \mathrm{H}, \mathrm{H}^{15}\right), 3.18\left(\mathrm{tm}, \mathrm{J}\left(\mathrm{H}^{2}, \mathrm{H}^{3}\right)=5.6 \mathrm{~Hz}, 2 \mathrm{H}, \mathrm{H}^{2}\right), 2.01-1.82(\mathrm{~m}$, $\left.3 \mathrm{H}, 2 \mathrm{H}^{18}+\mathrm{H}^{19}\right), 1.69\left(\mathrm{~m}, 1 \mathrm{H}, \mathrm{H}^{19^{\prime}}\right) .{ }^{13} \mathrm{C} \mathrm{NMR}\left(126 \mathrm{MHz}, \mathrm{CDCl}_{3}\right)$ : 
$\delta=150.6\left(\mathrm{~s}, \mathrm{C}^{10}\right), 147.0\left(\mathrm{dd},{ }^{1} \mathrm{~J}\left(\mathrm{C}^{5}, \mathrm{~F}^{5}\right)=243.2 \mathrm{~Hz},{ }^{3} \mathrm{~J}\left(\mathrm{C}^{5}, \mathrm{~F}^{7}\right)=10.8 \mathrm{~Hz}, \mathrm{C}^{5}\right)$, $145.6\left(\mathrm{dd},{ }^{1} \mathrm{~J}\left(\mathrm{C}^{7}, \mathrm{~F}^{7}\right)=241.4 \mathrm{~Hz},{ }^{3} \mathrm{~J}\left(\mathrm{C}^{7}, \mathrm{~F}^{5}\right)=10.5 \mathrm{~Hz}, \mathrm{C}^{7}\right), \quad 142.7\left(\mathrm{~s}, \mathrm{C}^{13}\right)$, 132.6(dd, $\left.{ }^{2} J\left(\mathrm{C}^{4 \mathrm{a}}, \mathrm{F}^{5}\right)=11.4 \mathrm{~Hz}, \quad \mathrm{C}^{4 \mathrm{a}}\right), \quad 130.8\left(\mathrm{dd}, \quad{ }^{2} \mathrm{~J}\left(\mathrm{C}^{8}, \mathrm{~F}^{7}\right)=11.3 \mathrm{~Hz}\right.$, $\left.\mathrm{C}^{8}\right), \quad 118.9\left(\mathrm{dd},{ }^{3} \mathrm{~J}\left(\mathrm{C}^{8 \mathrm{a}}, \mathrm{F}^{7}\right)=4.7 \mathrm{~Hz}, \mathrm{C}^{8 \mathrm{a}}\right), \quad 110.6\left(\mathrm{~s}, \mathrm{C}^{12}\right), \quad 109.5\left(\mathrm{t},{ }^{2} \mathrm{~J}\right.$ $\left.\left(\mathrm{C}^{6}, \mathrm{~F}^{7}\right) \approx 2 \mathrm{~J}\left(\mathrm{C}^{6}, \mathrm{~F}^{5}\right)=25.8 \mathrm{~Hz}, \mathrm{C}^{6}\right), 109.1\left(\mathrm{~d}, \mathrm{C}^{11}\right), 78.0\left(\mathrm{~d}, \mathrm{C}^{16}\right), 68.1\left(\mathrm{~s}, \mathrm{C}^{17}\right)$, $50.7\left(\mathrm{~d}, J\left(\mathrm{C}^{9}, \mathrm{~F}^{5}\right)=9.0 \mathrm{~Hz}, \mathrm{C}^{9}\right), 50.5\left(\mathrm{~d}, \mathrm{~J}\left(\mathrm{C}^{15}, \mathrm{~F}^{7}\right)=10.1 \mathrm{~Hz}, \mathrm{C}^{15}\right), 49.3\left(\mathrm{~s}, \mathrm{C}^{2}\right)$, $46.3\left(\mathrm{~s}, \mathrm{C}^{3}\right), 28.6\left(\mathrm{~s}, \mathrm{C}^{18}\right), 25.7\left(\mathrm{~s}, \mathrm{C}^{19}\right) \cdot{ }^{19} \mathrm{~F}$ NMR $\left(282 \mathrm{MHz}, \mathrm{CDCl}_{3}\right): \delta=-$ $124.0\left(\mathrm{~d}, \quad J\left(F^{5}, H^{6}\right)=12.0 \mathrm{~Hz}, 1 \mathrm{~F}, \mathrm{~F}^{5}\right),-128.0\left(\mathrm{dm}, \quad J\left(\mathrm{~F}^{7}, \mathrm{H}^{6}\right)=12.3 \mathrm{~Hz}\right.$, $\left.\mathrm{J}\left(\mathrm{F}^{7}, \mathrm{H}\right)=3 \mathrm{~Hz}, 1 \mathrm{~F}, \mathrm{~F}^{7}\right)$. HRMS(EI): $m / z[\mathrm{M}]^{+}$calcd for $\mathrm{C}_{18} \mathrm{H}_{2} 0 \mathrm{~F}_{2} \mathrm{~N}_{2} \mathrm{O}_{4} \mathrm{~S}$ : 398.1106; found: 398.1104 .

\section{4-Furfuryl-8-methylamino-5,7-difluoro-3,4-dihydro- $2 \mathrm{H}$ - benzo[1,4] thiazine-1,1-dioxide (3b)}

White solid (recrystallized from ethanol); yield: 0.72g (73\%); mp 87.3-88.0 ${ }^{\circ} \mathrm{C}$. IR (KBr): 3412, 3120, 2995, 2949, 1601, 1504, $1483,1429,1385,1306,1288,1244,1190,1146,1120,1036$, $1014,987,962,912,849,868,746,598,569,505,476 \mathrm{~cm}^{-1} .{ }^{1} \mathrm{H}$ NMR (500MHz, $\left.\mathrm{CDCl}_{3}\right): \delta=7.37\left(\mathrm{dd}, \mathrm{J}\left(\mathrm{H}^{13}, \mathrm{H}^{12}\right)=1.9 \mathrm{~Hz}, 1 \mathrm{H}, \mathrm{H}^{13}\right), 6.91$ $\left(\mathrm{t}, \mathrm{J}\left(\mathrm{H}^{6}, \mathrm{~F}^{5}\right) \approx \mathrm{J}\left(\mathrm{H}^{6}, \mathrm{~F}^{7}\right)=12.1 \mathrm{~Hz}, 1 \mathrm{H}, \mathrm{H}^{6}\right), 6.32\left(\mathrm{dd}, \mathrm{J}\left(\mathrm{H}^{12}, \mathrm{H}^{11}\right)=3.2 \mathrm{~Hz}\right.$, $\left.\mathrm{J}\left(\mathrm{H}^{12}, \mathrm{H}^{13}\right)=1.9 \mathrm{~Hz}, 1 \mathrm{H}, \mathrm{H}^{12}\right), 6.23\left(\mathrm{~d}, \mathrm{~J}\left(\mathrm{H}^{11}, \mathrm{H}^{12}\right)=3.2 \mathrm{~Hz}, 1 \mathrm{H}, \mathrm{H}^{11}\right), 4.93(\mathrm{~s}$, $\left.1 \mathrm{H}, \mathrm{H}^{14}[\mathrm{NH}]\right), 4.36\left(\mathrm{~s}, 2 \mathrm{H}, \mathrm{H}^{9}\right), 3.78\left(\mathrm{tm}, \mathrm{J}\left(\mathrm{H}^{3}, \mathrm{H}^{2}\right)=5.6 \mathrm{~Hz}, 2 \mathrm{H}, \mathrm{H}^{3}\right)$, $3.18\left(\mathrm{tm}, \mathrm{J}\left(\mathrm{H}^{2}, \mathrm{H}^{3}\right)=5.6 \mathrm{~Hz}, 2 \mathrm{H}, \mathrm{H}^{2}\right), 3.01\left(\mathrm{t}, 3 \mathrm{H}, \mathrm{J}\left(\mathrm{H}^{15}, \mathrm{~F}\right)=15.2 \mathrm{~Hz}, \mathrm{H}^{15}\right) \cdot{ }^{13} \mathrm{C}$ $\operatorname{NMR}\left(126 \mathrm{MHz}, \mathrm{CDCl}_{3}\right): \delta=150.5\left(\mathrm{~s}, \mathrm{C}^{10}\right), 147.0\left(\mathrm{dd},{ }^{1} \mathrm{~J}\left(\mathrm{C}^{5}, \mathrm{~F}^{5}\right)=243.9 \mathrm{~Hz}\right.$, $\left.3 \mathrm{~J}\left(\mathrm{C}^{5}, \mathrm{~F}^{7}\right)=10.7 \mathrm{~Hz}, \mathrm{C}^{5}\right), 145.6\left(\mathrm{dd},{ }^{1} \mathrm{~J}\left(\mathrm{C}^{7}, \mathrm{~F}^{7}\right)=241.2 \mathrm{~Hz},{ }^{3} \mathrm{~J}\left(\mathrm{C}^{7}, \mathrm{~F}^{5}\right)=10.4 \mathrm{~Hz}\right.$, $\left.\mathrm{C}^{7}\right), \quad 142.7\left(\mathrm{~s}, \mathrm{C}^{13}\right), \quad 133.6\left(\mathrm{dd},{ }^{2} \mathrm{~J}\left(\mathrm{C}^{4 \mathrm{a}}, \mathrm{F}^{5}\right)=11.3 \mathrm{~Hz}, \mathrm{C}^{4 \mathrm{a}}\right), \quad 130.7(\mathrm{dd}$, $\left.{ }^{2} \mathrm{~J}\left(\mathrm{C}^{8}, \mathrm{~F}^{7}\right)=11.3 \mathrm{~Hz}, \mathrm{C}^{8}\right), \quad 118.5\left(\mathrm{dd},{ }^{3} \mathrm{~J}\left(\mathrm{C}^{8 \mathrm{a}}, \mathrm{F}^{7}\right)=4.9 \mathrm{~Hz}, \mathrm{C}^{8 \mathrm{a}}\right), \quad 110.6(\mathrm{~s}$, $\left.\mathrm{C}^{12}\right), 109.7\left(\mathrm{t},{ }^{2} \mathrm{~J}\left(\mathrm{C}^{6}, \mathrm{~F}^{7}\right) \approx^{2} \mathrm{~J}\left(\mathrm{C}^{6}, \mathrm{~F}^{5}\right)=25.7 \mathrm{~Hz}, \mathrm{C}^{6}\right), 109.2\left(\mathrm{~d}, \mathrm{C}^{11}\right), 50.5(\mathrm{~d}$, $\left.\mathrm{J}\left(\mathrm{C}^{9}, \mathrm{~F}^{5}\right)=10.2 \mathrm{~Hz}, \mathrm{C}^{9}\right), 49.3\left(\mathrm{~s}, \mathrm{C}^{2}\right), 46.1\left(\mathrm{~s}, \mathrm{C}^{3}\right), 34.1\left(\mathrm{~d}, \mathrm{~J}\left(\mathrm{C}^{15}, \mathrm{~F}^{7}\right)=10.1 \mathrm{~Hz}\right.$, $\left.\mathrm{C}^{15}\right)$. ${ }^{19} \mathrm{~F}$ NMR $\left(282 \mathrm{MHz}, \mathrm{CDCl}_{3}\right): \delta=-127.1\left(\mathrm{~d}, \mathrm{~J}\left(\mathrm{~F}^{5}, \mathrm{H}^{6}\right)=12.0 \mathrm{~Hz}, 1 \mathrm{~F}\right.$, $\left.\mathrm{F}^{5}\right),-128.9\left(\mathrm{~m}, 1 \mathrm{~F}, \mathrm{~F}^{7}\right)$. HRMS(EI): $m / z[\mathrm{M}]^{+}$calcd for $\mathrm{C}_{14} \mathrm{H}_{14} \mathrm{~F}_{2} \mathrm{~N}_{2} \mathrm{O}_{3} \mathrm{~S}$ : 328.0682; found: 328.0688 .

\section{4-Benzyl-8-cyclopentanamino-5,7-difluoro-3,4-dihydro-2 $\mathrm{H}$ - benzo[1,4]thiazine-1,1-dioxide (5)}

Gray solid (recrystallized from acetone with hexane); yield: 0.88g (75\%); mp 99.1-99.5 C. IR (KBr): 3404, 3086, 3059, 2972, 2935, 2854, 1601, 1537, 1483, 1450, 1400, 1354, 1281, 1228, $1200,1120,1045,997,978,866,754,735,723,698,561,480 \mathrm{~cm}^{-}$ 1. ${ }^{1} \mathrm{H}$ NMR $\left(300 \mathrm{MHz}, \mathrm{CDCl}_{3}\right): \delta=7.39-7.30\left(\mathrm{~m}, 5 \mathrm{H}, \mathrm{H}_{\mathrm{ar}}[\mathrm{Ph}]\right), 6.92(\mathrm{t}$, $\left.\mathrm{J}\left(\mathrm{H}^{6}, \mathrm{~F}^{5}\right) \approx \mathrm{J}\left(\mathrm{H}^{6}, \mathrm{~F}^{7}\right)=12.6 \mathrm{~Hz}, 1 \mathrm{H}, \mathrm{H}^{6}\right), 5.03\left(\mathrm{~d}, 1 \mathrm{H}, \quad \mathrm{J}\left(\mathrm{H}^{14}, \mathrm{H}^{15}\right)=5.4 \mathrm{~Hz}\right.$,

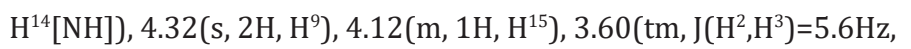
$\left.2 \mathrm{H}, \mathrm{H}^{2}\right), 3.28\left(\mathrm{tm}, \mathrm{J}\left(\mathrm{H}^{2}, \mathrm{H}^{3}\right)=5.6 \mathrm{~Hz}, 2 \mathrm{H}, \mathrm{H}^{2}\right), 1.91-1.89\left(\mathrm{~m}, 2 \mathrm{H}, \mathrm{H}^{16}\right), 1.84-$ $1.70\left(\mathrm{~m}, 2 \mathrm{H}, \mathrm{H}^{16}\right), 1.60-1.54\left(\mathrm{~m}, 4 \mathrm{H}, \mathrm{H}^{17}\right) .{ }^{13} \mathrm{C} \mathrm{NMR}\left(126 \mathrm{MHz}, \mathrm{CDCl}_{3}\right)$ : $\delta=146.4\left(\mathrm{dd},{ }^{1} \mathrm{~J}\left(\mathrm{C}^{7}, \mathrm{~F}^{7}\right)=244.0 \mathrm{~Hz},{ }^{3} \mathrm{~J}\left(\mathrm{C}^{7}, \mathrm{~F}^{5}\right)=10.6 \mathrm{~Hz}, \mathrm{C}^{7}\right), 145.6(\mathrm{dd}$, $\left.{ }^{1} \mathrm{~J}\left(\mathrm{C}^{5}, \mathrm{~F}^{5}\right)=240.8 \mathrm{~Hz},{ }^{3} \mathrm{~J}\left(\mathrm{C}^{5}, \mathrm{~F}^{7}\right)=10.6 \mathrm{~Hz}, \mathrm{C}^{5}\right), 137.0\left(\mathrm{~s}, \mathrm{C}^{10}\right), 132.2(\mathrm{dd}$, $\left.{ }^{2} \mathrm{~J}\left(\mathrm{C}^{4 \mathrm{a}}, \mathrm{F}^{5}\right)=3.2 \mathrm{~Hz}, \mathrm{C}^{4 \mathrm{a}}\right), 132.1\left(\mathrm{dd},{ }^{2} \mathrm{~J}\left(\mathrm{C}^{8}, \mathrm{~F}^{7}\right)=3.6 \mathrm{~Hz}, \mathrm{C}^{8}\right), 128.7\left(\mathrm{~s}, \mathrm{C}^{12}\right)$, $128.0\left(\mathrm{~d}, \mathrm{C}^{11}\right), 127.9\left(\mathrm{~s}, \mathrm{C}^{13}\right), 118.3\left(\mathrm{dd},{ }^{3} \mathrm{~J}\left(\mathrm{C}^{8 \mathrm{a}}, \mathrm{F}^{7}\right)=5.5 \mathrm{~Hz}, \mathrm{C}^{8 \mathrm{a}}\right), 109.7(\mathrm{t}$, $\left.{ }^{2} \mathrm{~J}\left(\mathrm{C}^{6}, \mathrm{~F}^{7}\right) \approx^{2} \mathrm{~J}\left(\mathrm{C}^{6}, \mathrm{~F}^{5}\right)=25.8 \mathrm{~Hz}, \mathrm{C}^{6}\right), 57.8\left(\mathrm{~d}, \mathrm{~J}\left(\mathrm{C}^{15}, \mathrm{~F}^{7}\right)=9.3 \mathrm{~Hz}, \mathrm{C}^{15}\right), 57.7(\mathrm{~d}$, $\left.\mathrm{J}\left(\mathrm{C}^{9}, \mathrm{~F}^{5}\right)=7.6 \mathrm{~Hz}, \mathrm{C}^{9}\right), 48.8\left(\mathrm{~s}, \mathrm{C}^{2}\right), 44.2\left(\mathrm{~s}, \mathrm{C}^{3}\right), 33.9\left(\mathrm{~d}, \mathrm{C}^{16}\right), 23.4(\mathrm{~s}$, $\left.\mathrm{C}^{17}\right) .{ }^{19} \mathrm{~F}$ NMR $\left(282 \mathrm{MHz}, \mathrm{CDCl}_{3}\right): \delta=-127.0\left(\mathrm{dd}, J\left(\mathrm{~F}^{7}, \mathrm{H}^{6}\right)=12.4 \mathrm{~Hz}\right.$,
$\left.J\left(F^{7}, H\right)=3.7 H z, 1 F, F^{7}\right),-128.9\left(d, J\left(F^{5}, H^{6}\right)=11.8 H z, 1 F, F^{5}\right)$. HRMS (EI): $m / z[\mathrm{M}]^{+}$calcd for $\mathrm{C}_{20} \mathrm{H}_{22} \mathrm{~F}_{2} \mathrm{~N}_{2} \mathrm{O}_{3} \mathrm{~S}$ : 392.1365; found: 392.1369 .

\section{Crystallographic Study}

XRD data were obtained on a Bruker Kappa Apex II CCD diffractometer using $\phi, \omega$ scans of narrow $\left(0.5^{\circ}\right)$ frames with Mo K $\alpha$ radiation $(\lambda=0.71073 \AA)$ and a graphite monochromator. The structures were solved by direct methods and refined by full-matrix least-squares method against all F2 in anisotropic approximation using the SHELX-97 programs set [21]. The $\mathrm{H}$ atoms positions were calculated with the riding model. Absorption corrections were applied empirically using Bruker SADABS programs [22]. The obtained crystal structures were analyzed for short contacts between non-bonded atoms using PLATON [23] and MERCURY [24] programs (Figure 3-5). In the analysis of shorted (as compared to the sum of the van der Waals radius) non-valent contacts, the values of the van der Waals radius proposed by Rowland and Taylor [25] were used (see SI).

\section{Crystallographic data for 2}

Deposition Number 2068933; Data Block Name: data_2_1687; Unit Cell Parameters: a 9.8958(5) b 12.5476(7) c 12.7746(8) P-1.

\section{Crystallographic data for $3 a$}

Deposition Number 2068934; Data Block Name: data_3a_695lt; Unit Cell Parameters: a 11.9170(7) b 9.0045(3) c 17.4951(10) $\mathrm{P} 21 / \mathrm{c}$.

\section{Crystallographic data for $3 \mathbf{b}$}

Deposition Number 2068935; Data Block Name: data_3b_ plv693; Unit Cell Parameters: a 8.7825(5) b 20.2301(11) c 8.0235(4) Pna21.

\section{Crystallographic data for $3 c$}

Deposition Number 2068936; Data Block Name: data_3c_ plt690; Unit Cell Parameters: a 9.6146(3) b 16.4749(7) c 11.7188(5) $\mathrm{P} 21 / \mathrm{n}$.

\section{Results}

The evaluation of antiarrhythmic properties of 8-tetrahydrofurfurylamino- (3a), 8-methylamino- (3b), and 8-cyclopentylamino- (3c) 4-furfuryl-5,7-difluoro-3,4-dihydro$2 \mathrm{H}$-benzo[1,4]thiazin-1,1-dioxide as well as 4-benzyl-8cyclopentylamino-5,7-difluoro-3,4-dihydro- $2 H$-benzo[1,4]thiazin1,1-dioxide (5) was performed on anesthetized mature male Wistar rats weighing 180-220g in groups of 10 animals. Among the obtained compounds (Figure 3), those marked in red and labeled as 3a, 3b, 3c, and 5 showed the highest activity. Agent 5's antiarrhythmic activity was not detectable in both models. The range of activity screening for these compounds was 0.05 to $5 \mathrm{mg} /$ $\mathrm{kg}$. The findings are presented in Table 1. 

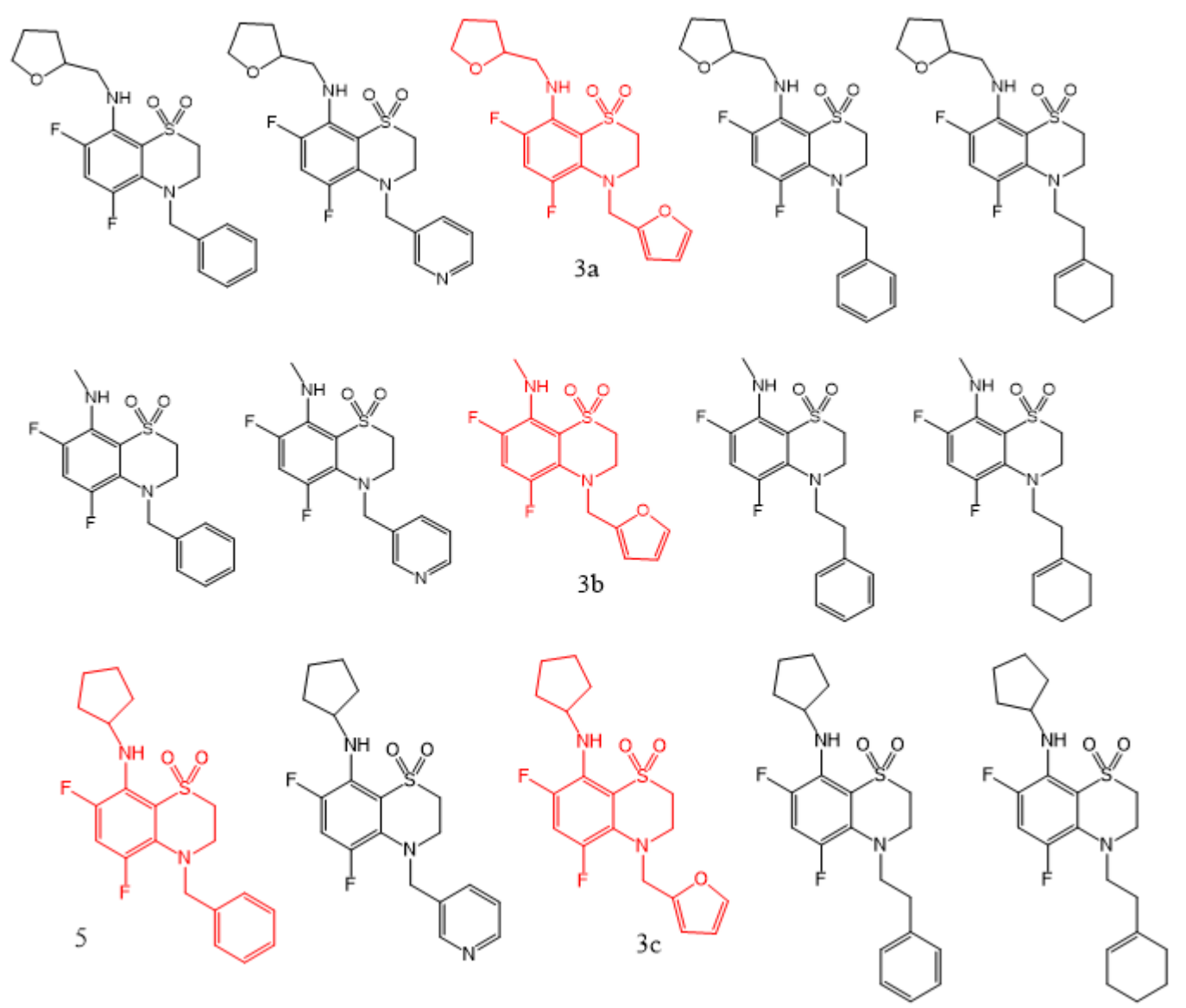

Figure 3: Derivatives of the benzothiazine series that were tested for antiarrhythmic properties in this study (active derivatives are highlighted in red).

Table 1: Antiarrhythmic activity of compounds $3 a, 3 b, 3 c$, and 5 .

\begin{tabular}{|c|c|c|c|}
\hline \multirow{2}{*}{ Agents } & \multirow{2}{*}{$\mathrm{mg} / \mathrm{kg}$} & \multicolumn{2}{|c|}{ The Percentage of Surviving Animals (\%) } \\
\hline & & Chloride Calcium Arrhythmia & Adrenaline Arrhythmia \\
\hline \multirow{3}{*}{$3 a$} & 5 & 50 & 50 \\
\hline & 0.5 & 100 & 50 \\
\hline & 0.05 & 0 & 90 \\
\hline \multirow{3}{*}{$3 b$} & 5 & 40 & 50 \\
\hline & 0.5 & 0 & 100 \\
\hline & 0.05 & 0 & 90 \\
\hline \multirow{3}{*}{$3 c$} & 5 & 0 & 100 \\
\hline & 0.5 & 0 & 100 \\
\hline & 0.05 & 0 & 0 \\
\hline \multirow{2}{*}{5} & 5 & 0 & 0 \\
\hline & 0.5 & 0 & 0 \\
\hline \multirow{2}{*}{ Metoprolol } & 3 & 100 & 100 \\
\hline & 0.15 & 0 & 50 \\
\hline
\end{tabular}

As readers can see in the Table 1 , these derivatives had the strongest antiarrhythmic effect on epinephrine-induced arrhythmia. Agents $3 \mathrm{a}$ and $3 \mathrm{~b}$ yielded $90 \%$ survival of the animals at doses of $0.05 \mathrm{mg} / \mathrm{kg}$, which is 60 -fold lower than that of a comparison drug, metoprolol. Agent $3 \mathrm{c}$ showed high efficacy at a dose of $0.5 \mathrm{mg} / \mathrm{kg}$, which is 6 -fold lower than that of the reference 
drug. By contrast, 3c exerted no action on calcium chloride-induced arrhythmia, whereas agent $3 \mathrm{a}$ manifested high activity at the $0.5 \mathrm{mg} / \mathrm{kg}$ dose. Agent $3 \mathrm{~b}$ at $5 \mathrm{mg} / \mathrm{kg}$ showed efficacy in $40 \%$ of the studied rats. Therefore, it can be concluded that compounds $3 \mathrm{a}$ and $3 \mathrm{~b}$ (derivatives of fluorinated 4-furfuryl-3,4-dihydro- $2 \mathrm{H}$-benzo[1,4] thiazine-1,1-dioxides) in doses of $0.05,0.5$, and $5 \mathrm{mg} / \mathrm{kg}$ exert strong antiarrhythmic effects on calcium chloride and epinephrine models of arrhythmia. The compounds have a dose-dependent effect on both models. At the same time, it can be assumed that the presence of the furfuryl moiety at the $4^{\text {th }}$ position plays a key role in the antiarrhythmic action of the benzothiazine derivatives.

The absence of the cyclopentylamine substituent at position
8 enhances the antiarrhythmic effect on calcium chloride-induced arrhythmia. The observed strong antiarrhythmic effect on epinephrine-induced arrhythmia suggests that these derivatives can affect blood pressure in rats. Investigation of the hypertensive properties of compounds 3a-5 was performed on anesthetized (sodium thiopental at $0.12 \mathrm{mg} / \mathrm{kg}$ intraperitoneally) mature male Wistar rats weighing 180-220g. Drug preparations were injected at $5 \mathrm{mg} / \mathrm{kg}$ into the femoral vein per 10 animals in the groups. Blood pressure was measured in acute settings via insertion of a cannula into the carotid artery. It turned out that the intravenous administration of these agents causes a gradual increase in blood pressure within $5 \mathrm{~min}$ (Table 2).

Table 2: The influence of agents $3 a, 3 b, 3 c$, and 5 on rat ECG parameters.

\begin{tabular}{|c|c|c|c|c|c|c|c|c|c|c|c|c|}
\hline \multirow{3}{*}{ Agents } & \multicolumn{12}{|c|}{ ECG parameters before and after administration of an agent in dose $5 \mathrm{mg} / \mathrm{kg}$} \\
\hline & \multicolumn{2}{|c|}{ QRS (ms) } & \multicolumn{2}{|c|}{ ST (ms) } & \multicolumn{2}{|c|}{ QT (ms) } & \multicolumn{2}{|c|}{ Ra $(\mathrm{mV})$} & \multicolumn{2}{|c|}{ Ta $(\mathrm{mV})$} & \multicolumn{2}{|c|}{$\mathrm{Pa}(\mathrm{mV})$} \\
\hline & Before & After & Before & After & Before & After & Before & After & Before & After & Before & After \\
\hline \multirow{2}{*}{$3 a$} & $0.011 \pm$ & $0.011 \pm$ & $0.018 \pm$ & $0.018 \pm$ & $0.029 \pm$ & $0.029 \pm$ & $1460.7 \pm$ & $1422.9 \pm$ & $269.6 \pm$ & $291.3 \pm$ & $270.5 \pm$ & $279.05 \pm$ \\
\hline & 0.0008 & 0.0005 & 0.002 & 0.001 & 0.001 & 0.0009 & 277.3 & 245.5 & 17.1 & 65.3 & 37.6 & 38.2 \\
\hline \multirow{2}{*}{$3 b$} & $0.011 \pm$ & $0.011 \pm$ & $0.016 \pm$ & $0.017 \pm$ & $0.027 \pm$ & $0.028 \pm$ & $1280.3 \pm$ & $1256.3 \pm$ & $228.3 \pm$ & $233.5 \pm$ & $269.1 \pm$ & $263.6 \pm$ \\
\hline & 0.0012 & 0.002 & 0.005 & 0.004 & 0.005 & 0.002 & 302.3 & 232.2 & 70.03 & 34.7 & 32.7 & 26.05 \\
\hline \multirow{3}{*}{$3 c$} & $0.013 \pm$ & $0.013 \pm$ & $0.0158 \pm$ & 0.015 & 0.027 & 0.027 & 1206.4 & $1182.319 \pm$ & $267.6 \pm$ & $302.2648 \pm$ & & \\
\hline & 0.0013 & 0.0018 & 0.003 & \pm 0.003 & \pm 0.0035 & \pm 0.004 & \pm 220.2 & 209.15 & 47.4 & 55.9 & & 46.15 \\
\hline & & & & & & & & & & & $\mathrm{P}=0.001$ & $\mathrm{P}=0.0028$ \\
\hline \multirow{3}{*}{5} & $0.012 \pm$ & $0.012 \pm$ & $0017+$ & 0017 & 0028 & 0,028 & 13124 & 125 & 204 & 2908 & $270.8 \pm$ & $209.20 \pm$ \\
\hline & 0008 & & ?ח0? & 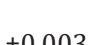 & +0002 & +0003 & $+190 ?$ & 18015 & 384 & & 35.1 & 37.8 \\
\hline & & & & & & & & & & & $\mathrm{P}=0.0025$ & $P=0.0063$ \\
\hline
\end{tabular}

\pm shows the spread of the mean square error from the mean data.

The most pronounced increase in blood pressure was observed after treatment with either agent $3 \mathrm{c}$ or 5: within $5 \mathrm{~min}$, blood pressure increased by $34 \%$ and $29 \%$, respectively, relative to initial blood pressure. After the administration of either $3 \mathrm{a}$ or $3 \mathrm{~b}$, blood pressure increased by only $7 \%$. The effect lasted more than $30 \mathrm{~min}$. Administration of the same volume of saline did not cause a change in blood pressure in the rats. The data indicated that the presence of a cyclopentylamine moiety at position 8 significantly enhances the hypertensive effect.

The results implied that these agents could affect an ECG of the rats and thus may have adverse effects (toxicity) in the clinic. Therefore, we carried out a toxicological analysis to determine LD50 and measured the main ECG parameters after the administration of these agents at $5 \mathrm{mg} / \mathrm{kg}$ per 10 animals in the groups (Table 3 ).

Acute toxicity was determined in white outbred mice weighing 18-22g after oral administration at 50 to $1000 \mathrm{mg} / \mathrm{kg}$. Parameters of acute toxicity were calculated according to the Kerber method. The LD50 of compounds $3 \mathrm{a}-\mathrm{c}$ and 5 was found to be greater than $1000 \mathrm{mg} / \mathrm{kg}$. Analysis of the main ECG parameters revealed complete absence of changes after the administration of agent $3 \mathrm{a}$ or $3 \mathrm{~b}$ and a significant increase in $\mathrm{P}$ wave amplitude for agents $3 \mathrm{c}$ and 5. This increase was within a normal physiological range (Table 2).

\section{The experiment on the Isolated Atrium (Organ Bath ex situ)}

In this experiment, the mechanism of action of the studied agents was investigated via the addition of epinephrine or calcium chloride to the aerated solution bathing a rat atrium pretreated with $3 \mathrm{a}, 3 \mathrm{~b}$, or $3 \mathrm{c}$ at $10^{-3} \mathrm{mmol} / \mathrm{L}$ (Table 3 ).

After the treatment with compound $3 \mathrm{~b}$ or $3 \mathrm{c}$ (Table 3), there was a significant increase in the amplitude of atrial contractions. The same increase in the amplitude of contractions was observed with the introduction of epinephrine or calcium chloride after the start of treatment with $3 a, 3 b$, or 3c (Table 3, Figures 4-6). These findings suggested that benzothiazine-type drugs are neither $\beta$-adrenergic receptor blockers nor calcium channel blockers. 
Table 3: Amplitude and frequency of contractions before and after administration of drugs 3a-c and substances that cause arrhythmia. ${ }^{*} p<0.05$.

\begin{tabular}{|c|c|c|c|c|c|c|c|c|c|c|c|c|c|}
\hline \multirow{3}{*}{ Agents } & \multirow{3}{*}{$\begin{array}{c}\text { Dose } \\
\text { (mmol/L) }\end{array}$} & \multicolumn{4}{|c|}{ Beginning } & \multicolumn{4}{|c|}{$\begin{array}{l}\text { Introduction of epinephrine after the } \\
\text { start of treatment with the drug }\end{array}$} & \multicolumn{4}{|c|}{$\begin{array}{l}\text { Introduction of calcium chloride after } \\
\text { the start of treatment with the drug }\end{array}$} \\
\hline & & \multicolumn{2}{|c|}{ Amplitude Y } & \multicolumn{2}{|c|}{$\begin{array}{l}\text { Frequency of } \\
\text { contractions X }\end{array}$} & \multicolumn{2}{|c|}{ Amplitude } & \multicolumn{2}{|c|}{$\begin{array}{l}\text { Frequency of } \\
\text { contractions }\end{array}$} & \multicolumn{2}{|c|}{ Amplitude } & \multicolumn{2}{|c|}{$\begin{array}{l}\text { Frequency of } \\
\text { contractions }\end{array}$} \\
\hline & & Before & After & Before & After & Before & After & Before & After & Before & After & Before & After \\
\hline $3 a$ & 10-Mar & $\begin{array}{c}0.085 \pm \\
0.008\end{array}$ & $\begin{array}{l}0.09 \pm \\
0.016\end{array}$ & $0.1 \pm 0$ & $\begin{array}{c}0.1 \pm \\
0\end{array}$ & $\begin{array}{c}0.078 \pm \\
0.0155^{*}\end{array}$ & $\begin{array}{l}0.108 \pm \\
0.0155\end{array}$ & $0.1 \pm 0$ & $\begin{array}{c}0.1 \pm \\
0\end{array}$ & $\begin{array}{c}0.12 \pm \\
0^{*}\end{array}$ & $\begin{array}{l}0.156 \pm \\
0.001 *\end{array}$ & $\begin{array}{c}0.1 \pm \\
0\end{array}$ & $0.1 \pm 0$ \\
\hline $3 b$ & 10-Mar & $0.04^{*}$ & $\begin{array}{c}0.06 \pm \\
0.0115^{*}\end{array}$ & $0.1 \pm 0$ & $\begin{array}{c}0.1 \pm \\
0\end{array}$ & $\begin{array}{l}0.105 \pm \\
0.0158^{*}\end{array}$ & $\begin{array}{l}0.177 \pm \\
0.017 *\end{array}$ & $0.1 \pm 0$ & $\begin{array}{c}0.1 \pm \\
0\end{array}$ & $\begin{array}{l}0.123 \pm \\
0.009^{*}\end{array}$ & $\begin{array}{c}0.32 \pm \\
0.045^{*}\end{array}$ & $\begin{array}{c}0.1 \pm \\
0\end{array}$ & $0.1 \pm 0$ \\
\hline $3 c$ & 10-Mar & $\begin{array}{c}0.164 \pm \\
0.0295^{*}\end{array}$ & $\begin{array}{c}0.221 \pm \\
0.0213^{*}\end{array}$ & $\begin{array}{l}0.1 \\
\pm 0\end{array}$ & $\begin{array}{c}0.1 \pm \\
0\end{array}$ & $\begin{array}{c}0.211 \pm \\
0.0251^{*}\end{array}$ & $\begin{array}{l}0.286 \pm \\
0.005^{*}\end{array}$ & $0.1 \pm 0$ & $\begin{array}{c}0.1 \pm \\
0\end{array}$ & $\begin{array}{l}0.108 \pm \\
0.015^{*}\end{array}$ & $\begin{array}{c}0.13 \pm \\
0.018^{*}\end{array}$ & $\begin{array}{c}0.1 \pm \\
0\end{array}$ & $0.1 \pm 0$ \\
\hline
\end{tabular}

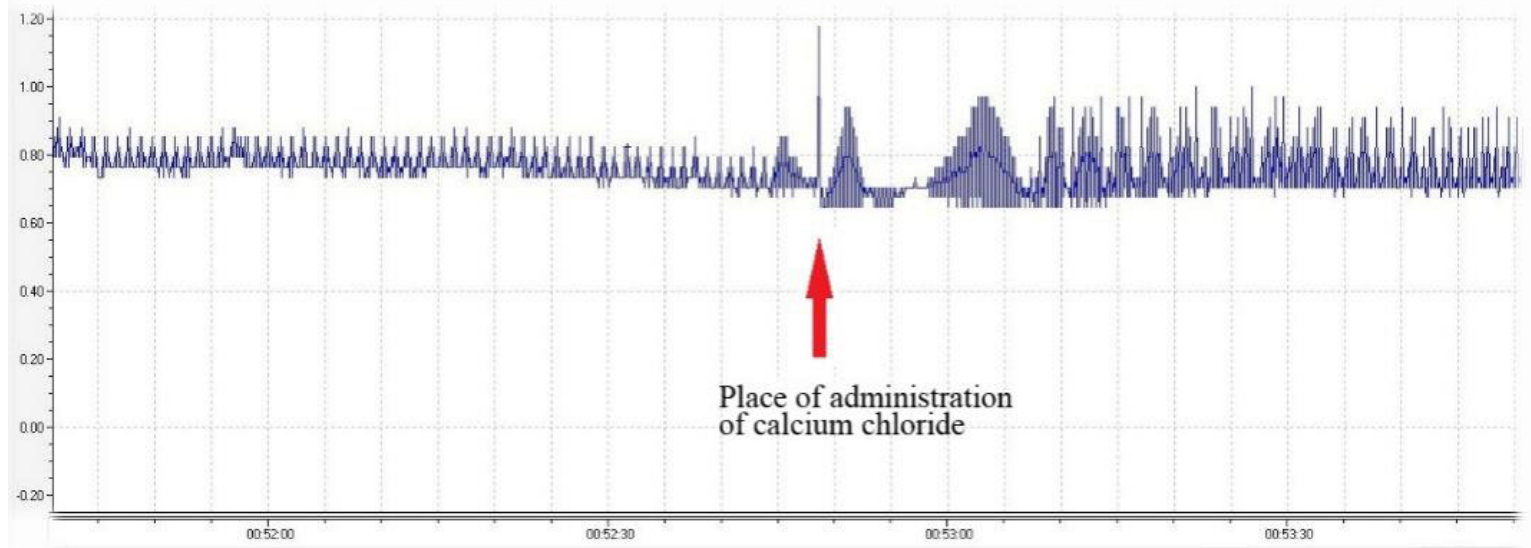

Figure 4: Atrial contractions before and after administration of calcium chloride during treatment with drug 3a.

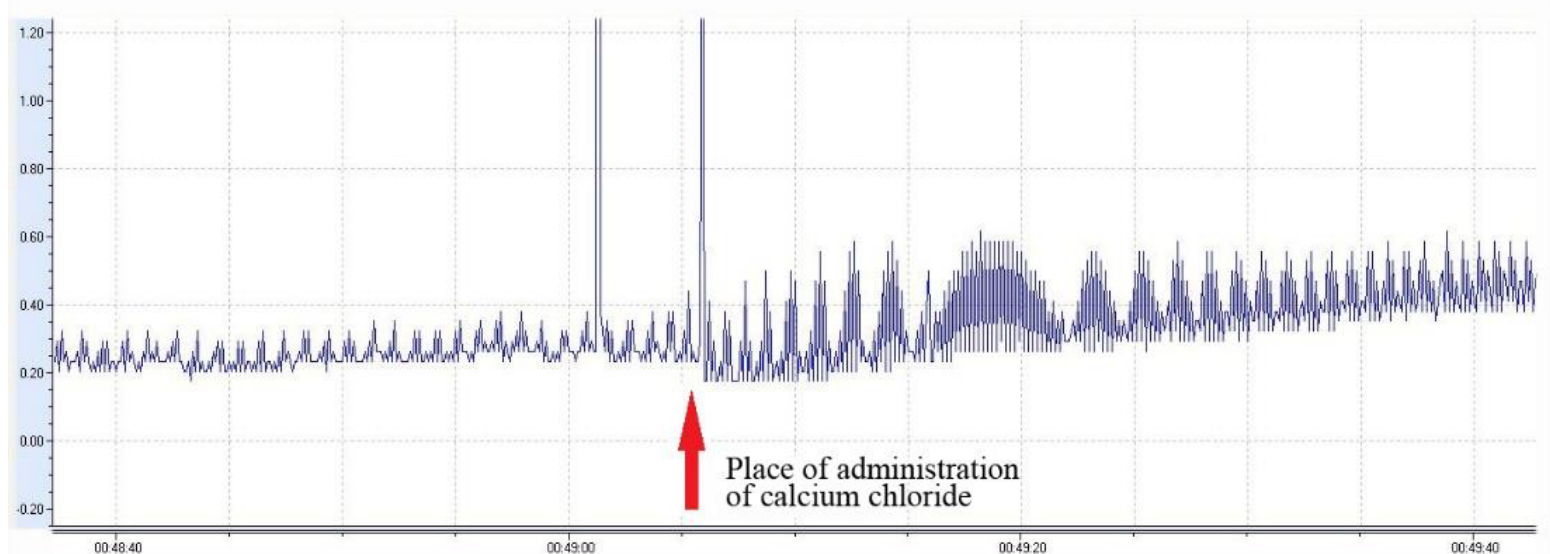

Figure 5: Atrial contractions before and after administration of calcium chloride during treatment with drug 3b. 


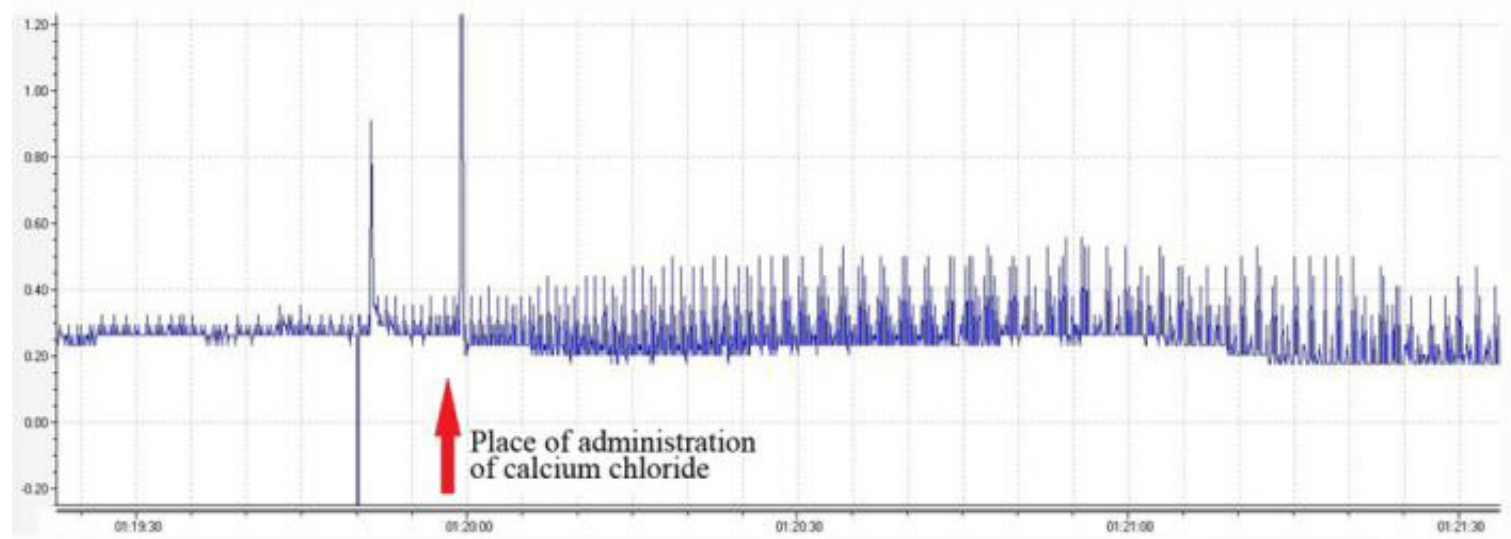

Figure 6: Atrial contractions before and after administration of calcium chloride during treatment with drug 3c.

\section{Discussion}

From our results, it can be concluded that compounds 3a, $3 \mathrm{~b}$, and $3 \mathrm{c}$ (derivatives of fluorinated 4-furfuryl-3,4-dihydro- $2 \mathrm{H}$ benzo[1,4]thiazine-1,1-dioxides) at doses of $0.05,0.5$, and $5 \mathrm{mg} / \mathrm{kg}$ have high antiarrhythmic activity in models of calcium chlorideinduced and epinephrine-induced arrhythmia. The compounds had a dose-dependent effect on both models. It can be assumed that the presence of the furfuryl group at position 4 (Figure 7) plays a crucial part in the antiarrhythmic properties of benzothiazine derivatives. The oxygen located in the furfuryl moiety is an acceptor with respect to nitrogen and draws on the electron density, thereby probably making the nitrogen atom more reactive and increasing the affinity of the ligand-receptor interaction.
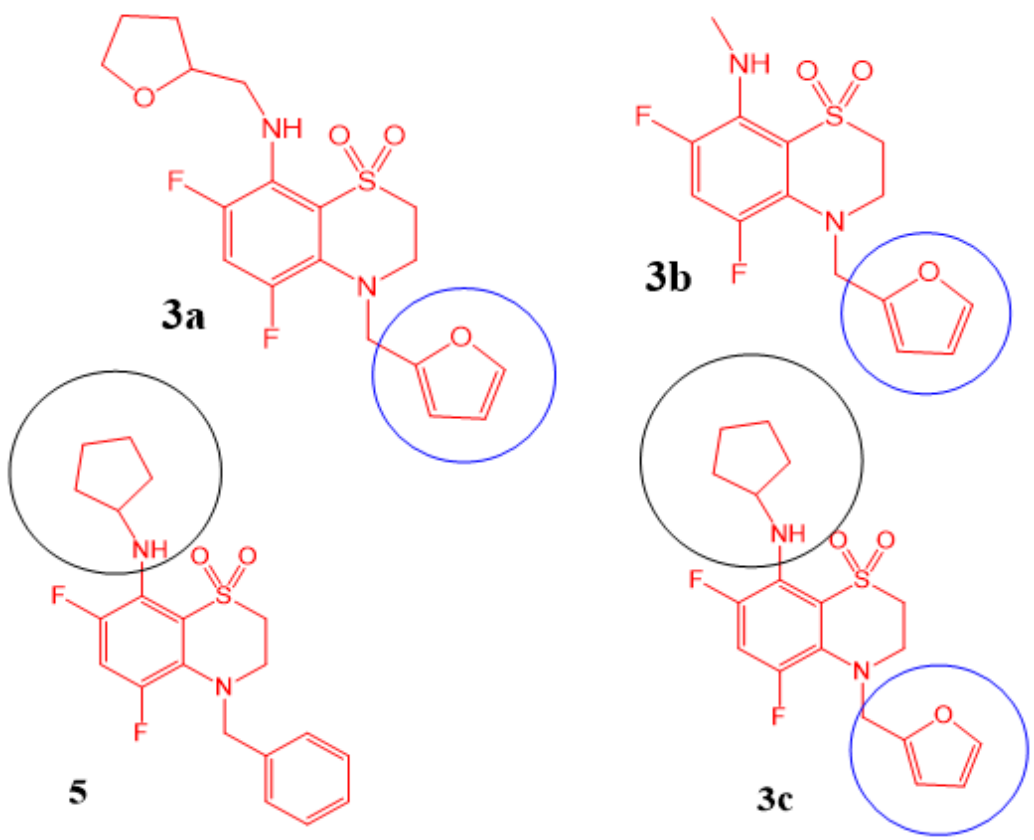

Figure 7: Structure of fluorinated 3,4-dihydro-2H-1,4-benzothiazin-1,1-dioxide derivatives 3a,b,c and 5.

The strong antiarrhythmic impact on epinephrine-induced arrhythmia suggests that these derivatives may affect blood pressure. As described in the Results section, agents $3 c$ and 5 had the most pronounced hypertensive effect. Agents $3 c$ and 5 share a similarity: the presence of a cyclopentylamine moiety at position 8 (Figure 7). The cyclopentane ring may make the nitrogen atom more energetically favorable during an interaction with blood pressure-influencing receptors. On the other hand, in agent $3 c$, there is no furfuryl group at the $4^{\text {th }}$ position and therefore no antiarrhythmic effect. Thus, our findings show that the presence of the cyclopentylamine moiety at position 3a significantly increases the hypertensive effect. 
Our assay of cardiotoxicity of the benzothiazine derivatives revealed no adverse effects on ECG parameters in the rats. The toxicity of antiarrhythmic drugs is the main reason for the need for new drugs of this class. Studies on the toxicity of benzothiazine derivatives indicate that these agents have class 3 toxicity (our study did not address this question). Consequently, they are promising therapies for arrhythmias of different etiologies as well as for a gradual increase of blood pressure in patients with hypotensive disorders.

Determination of possible mechanisms of action on the isolated atrium was performed in an organ bath. Working on an isolated atrium allows to identify an independent rhythm driver whose contractions are based on the passage of calcium through calcium channels, and which has $\beta$-adrenoreceptors that are sensitive to catecholamines. Thus, the addition of calcium to the bath with the atrium causes an increase in contraction amplitude, whereas the addition of epinephrine increases the frequency and amplitude. As a result, the blockage of calcium channels and $\beta$-adrenergic receptors with drugs should block these changes. As demonstrated in our experiment, the introduction of calcium chloride or epinephrine during the treatment with each of our drugs leads to an increase in the amplitude of contraction. Therefore, they are neither blockers of calcium channels nor $\beta$-adrenoceptor blockers.

\section{Conclusion}

High antiarrhythmic activity of $3 a, 3 b$, and $3 c$, which are derivatives of the benzothiazine series, was demonstrated, and a "monotonic" hypertensive effect was documented for 5 and 3c. Evaluation of the influence of compounds $3 \mathrm{a}, 3 \mathrm{~b}$, and $3 \mathrm{c}$ on the main ECG parameters in rats detected no cardiotoxic effects. According to our results on the rat atria in the organ bath, it can be assumed that $3 \mathrm{a}, 3 \mathrm{~b}$, and $3 \mathrm{c}$ are neither calcium channel blockers nor $\beta$-adrenoceptor blockers. Consequently, the research on the mechanism of action of these compounds should be continued.

\section{Acknowledgment}

The authors thank the Chemical Service Center SB RAS for spectral and analytical measurements, and government program AAAA-A21-121011490015-1 for aid in the preparation of the manuscript. The work on the synthesis of new polyfluorinated heterocycles was supported by Ministry of Science and Higher Education of the Russian Federation (Project AAAA-A21-121011490017-5).

\section{References}

1. Han H, Chao H, Guerra A, Sosa A, Christopoulos G, et al. (2015) Evolution of the american college of cardiology/american heart association clinical guidelines. J Am Coll Cardiol 65(25): 2726-2734.
2. Demirovic J, Myerburg RJ (1994) Epidemiology of sudden coronary death: an overview. Prog Cardiovasc Dis 37(1): 39-48.

3. Zipes DP, Wellens HJ (2000) What have we learned about cardiac arrhythmias? Circulation 102(20 Suppl 4): 52-57.

4. Ideker RE, Walcott GP, Epstein AE, Plumb VI, Kay N (2005) Ventricular fibrillation and defibrillation-What are the major unresolved issues? Hear Rhythm 2(5): 555-558.

5. Chattipakorn N, Ideker RE (2003) Delayed afterdepolarization inhibitor: a potential pharmacologic intervention to improve defibrillation efficacy. J Cardiovasc Electrophysiol 14(1): 72-75.

6. Mar PL, Raj SR (2018) Orthostatic hypotension for the cardiologist. Curr Opin Cardiol 33(1): 66-72.

7. Joseph A, Wanono R, Flamant M, Petiot EV (2017) Orthostatic hypotension: A review. Nephrol Ther 13 Suppl 1: S55-S67.

8. Goldstein DS, Robertson D, Esler M, Straus SE, Eisenhofer G (2002) Dysautonomias: clinical disorders of the autonomic nervous system. Ann Intern Med 137(9): 753-763.

9. Ricci F, Caterina RD, Fedorowski A (2015) Orthostatic hypotension: epidemiology, prognosis, and treatment. J Am Coll Cardiol 66(7): 848860.

10. Sutton R (2013) Clinical classification of syncope. Prog Cardiovasc Dis 55(4): 339-344.

11. Fringuelli R, Milanese L, Schiaffella F (2005) Role of 1,4-benzothiazine derivatives in medicinal chemistry. Mini Rev Med Chem 5(12): 10611073.

12. Ajani OO (2012) Functionalized 1,4-benzothiazine: a versatile scaffold with diverse biological properties. Arch Pharm (Weinheim) 345(11): 841-851.

13. Litvak VV, Shteimgarts KAS (2009) Synthesis of $\beta$-functionalized ethyl polyfluoroaryl sulfides, sulfoxides and sulfones underlain by pentafluorobenzoic acid. Rus J Org Chem 45: 1637-1643.

14. Kondratyev AS, Shteingarts VD, Litvak VV, Tretyakov EV, Tkachev AV (2017) Domino reaction of (2-haloethyl)polyfluorophenyl sulfides, sulfoxides, and sulfones with ammonia or amines: one-pot synthesis of 3,4-dihydro-2H-1,4-benzothiazines polyfluorinated at the benzene ring and the corresponding 1-oxides and 1,1-dioxides. Chem Heterocycl Compd 7: 1350-1361.

15. Politanskaya L, Tretyakov E (2018) p-Toluenesulfonic acid induced conversion of fluorinated trimethylsilylethynylanilines into aminoacetophenones: Versatile precursors for the synthesis of benzoazaheterocycles. Synthesis (Stuttg) 50: 555-564.

16. Politanskaya L, Rybalova T, Zakharova O, Nevinsky G, Tretyakov E (2018) p-Toluenesulfonic acid mediated one-pot cascade synthesis and cytotoxicity evaluation of polyfluorinated 2-aryl-2,3-dihydroquinolin-4ones and their derivatives. J Fluorine Chem 211: 129-140.

17. Politanskaya LV, Chuikov IP, Shteingarts VD (2013) Synthesis of indoles with a polyfluorinated benzene ring. Tetrahedron 69: 8477-8486.

18. Politanskaya LV, Shteingarts VD, Tretyakov EV (2016) General and efficient synthesis of polyfluorinated 2-aminotolans and 2-arylindoles. J Fluorine Chem 188: 85-98.

19. Politanskaya LV, Chuikov IP, Tretyakov EV, Shteingarts VD, Ovchinnikova LP, et al. (2015) An effective two-step synthesis, fluorescent properties, antioxidant activity and cytotoxicity evaluation of benzene-fluorinated 2,2-dimethyl-2,3-dihydro-1H-quinolin-4-ones. J Fluorine Chem 178: 142-153. 
20. Politanskaya L, Tretyakov E (2020) Directed synthesis of fluorine containing 2,3-dihydrobenzo[b][1,4] oxathiine derivatives from polyfluoroarenes. J Fluorine Chem 236: 109592.

21. (1997) GMS programs for crystal structure analysis, University of Göttingen, Germany.

22. SADABS (2008) Bruker AXS, Madison, WI, USA.

23. Sperk AL (2001) Single-crystal structure validation with the program PLATON. J Appl Cryst 36(1): 7-13.
24. Macrae CF, Edgington PR, McCabe P, Pidcock E, Shields GP, et al. (2006) Mercury: visualization and analysis of crystal structures. J Appl Crystallogr 39: 453-457.

25. Rowland RS, Taylor R (1996) Intermolecular nonbonded contact distances in organic crystal structures: comparison with distances expected from van der Waals radii. J Phys Chem 100(18): 7384-7391. 